\title{
The non-thermal broadband spectral energy distribution of radio galaxies
}

\author{
Gustavo E. Romero ${ }^{1,2, \dagger}$ \\ ${ }^{1}$ Instituto Argentino de Radioastronomía (IAR, CCT La Plata, CONICET), C.C.5, (1894) \\ Villa Elisa, Buenos Aires, Argentina \\ ${ }^{2}$ Facultad de Ciencias Astronómicas y Geofísicas, Universidad Nacional de La Plata, Paseo del \\ Bosque s/n, 1900 La Plata, Argentina \\ email: romero@fcaglp.unlp.edu.ar romero@iar-conicet.gov.ar
}

\begin{abstract}
I present a model for the non-thermal production of electromagnetic radiation in the jets of radio galaxies. The model goes far beyond the simple one-zone models usually applied to these sources. The transport equation is solved in the co-moving frame of the jet, taken into account the inhomogeneous structure of the outflow. Energy distributions for all types of particles are then obtained in a self-consistent way, including protons, electrons, and secondaries. The spectral energy distribution resulting from all relevant radiative processes is computed, including synchrotron radiation, relativistic Bremsstrahlung, proton-proton collisions and subsequent decays, photo-meson production, radiation from pairs formed by photon absorption and injection from decays, as well as direct pair production. Absorbing fields in the host galaxy are considered when computing the final SED. The model is applied to Centaurus A and compared with the available multi-wavelength data.
\end{abstract}

Keywords. Galaxies: active, galaxies: jets, gamma rays: theory, radiation mechanisms: nonthermal.

\section{Introduction}

Radio galaxies can display electromagnetic emission across the entire spectrum, reaching in some cases muti-TeV energies. The most active radio galaxies are classified as Faranoff-Riley type I (FR I) and Faranoff-Riley type II (FR II). The former are intrinsically weaker sources, with extended radio lobes and plumes. The latter are powerful sources, with higher accretion rates and more luminous jets, that terminate in bright hot spots. Nearby FR I radio galaxies such as Centaurus A (Cen A) and M87 have been recently detected at very high-energies. These galaxies show significant activity in the central region, with strong variability reported at different wavelengths. This variability indicates that a significant part of the non-thermal emission is produced in the inner part of the relativistic jets, close to the central source. In what follows I present the outlines of a model that can explain the broadband nuclear emission of active radio galaxies and I show an application of such a model to the specific case of Cen A. For additional details the reader is referred to Reynoso et al. (2011).

\section{Basics of the model}

I assume that the jet is launched from the black hole's ergosphere, being completely formed at a distance $z_{0}=50 R_{\mathrm{g}}$, and with an initial bulk Lorentz factor $\Gamma_{0}$. Equipartition

\section{$\dagger$ Member of CONICET.}


holds between the magnetic and kinetic energy at the base of the jet. This is required if the plasma is set in motion by magneto-centrifugal effects. The kinetic power is given by $L_{\mathrm{j}}^{(\mathrm{kin})}=\frac{q_{\mathrm{j}}}{2} L_{\mathrm{Edd}}$, where $L_{\mathrm{Edd}}$ is the Eddington luminosity and $0<q_{\mathrm{j}}<1$ is a numerical factor. The jet is modeled as a cone with an half-opening angle $\xi$. Most of its content is thermal plasma. The value of the magnetic field at the position $z_{0}$ in jet is

$$
B_{0}=\sqrt{\frac{8 L_{\mathrm{j}}^{(\mathrm{kin})}}{\left[r_{\mathrm{j}}\left(z_{0}\right)\right]^{2} v_{\mathrm{b}}}},
$$

where $r_{\mathrm{j}}\left(z_{0}\right)=z_{0} \tan \xi$ is the jet radius at $z_{0}$.

The jet magnetic energy is gradually converted into bulk kinetic energy and the field decreases as the flow accelerates. The later is parametrized by

$$
B(z)=B_{0}\left(\frac{z_{0}}{z}\right)^{m}
$$

with $m \in\{1,2\}$. If all the magnetic energy is transformed into bulk kinetic energy, the Lorentz factor of the jet increases with $z$ approximately as

$$
\Gamma_{\mathrm{b}}(z) \approx 1+\frac{B_{0}^{2} z_{0}^{2} \tan ^{2} \xi}{8 \dot{m}_{\mathrm{j}} c}\left[2-\left(\frac{z_{0}}{z}\right)^{2 m-2}\right] .
$$

In turn, a fraction $q_{\text {rel }}$ of the bulk kinetic power is transformed into highly relativistic particles in a region that starts at $z_{\text {acc }}$. In this region the bulk kinetic energy is well below the magnetic one (sub-partition condition), and shocks can develop. Both primary electrons and protons are assumed to be injected at $z_{\text {acc }}$, over a length $\Delta z$ along the jet, with a power law distribution in the frame of the jet,

$$
Q_{e, p}^{\prime}\left(z^{\prime}, E^{\prime}\right)=\frac{K_{e, p}}{4 \pi}\left(\frac{z_{\mathrm{acc}}^{\prime}}{z^{\prime}}\right)\left(\frac{E^{\prime}}{m_{i} c^{2}}\right)^{-s} e^{-E^{\prime} / E_{\mathrm{max}}^{\prime}},
$$

in units of $\left(\mathrm{GeV}^{-1} \mathrm{~s}^{-1} \mathrm{~cm}^{-3} \mathrm{sr}^{-1}\right)$.

The constants $K_{e, p}$ are fixed by normalizing the injected power as

$$
L_{e, p}^{\prime}=\int_{V^{\prime}} d V^{\prime} \int_{E_{\min }^{\prime}} d E^{\prime} E^{\prime} Q^{\prime}\left(z^{\prime}, E^{\prime}\right) .
$$

The acceleration mechanism might be diffusive shock acceleration, but other possibilities cannot be ruled out.

The maximum energies of the injected particles are determined from the balance between the acceleration rate and the cooling rates. The proton to electron ratio at the injection point is given by the parameter $a=L_{p}^{\prime} / L_{e}^{\prime}$, where $L_{e}^{\prime}+L_{p}^{\prime}=q_{\mathrm{rel}} L_{\mathrm{j}}^{(\mathrm{kin})}$.

The main cooling processes are synchrotron emission for electrons and protons, and $p p$, $p \gamma$ interactions and adiabatic cooling for protons. Inverse Compton (IC) interactions with synchrotron photons in the jet are also considered. The expression for the acceleration rate is: $t_{\text {acc }}^{-1}=\eta c e B^{\prime}(z) / E^{\prime}$. For the other rates the reader can see Romero \& Vila (2008) and Vila \& Romero (2010).

The steady-state particle distributions $N_{i}(E, z)$ in the jet can be found by solving a 1-dimensional transport equation with cooling and convection along the jet, with a bulk velocity $v \approx c$, as seen in the observer frame:

$$
v \frac{\partial N_{i}}{\partial z}+\frac{\left.\partial\left[b_{i}(z, E) N_{i}\right)\right]}{\partial E}+\frac{N_{i}}{T_{\mathrm{dec}}(E)}=Q_{i}(z, E) .
$$

This equation is solved first for electrons, assuming no IC cooling. This first 
approximation to $N_{e}(z, E)$ is then iteratively improved taking into account also the IC cooling rate $t_{I C}^{-1}$ to solve the transport equation. The distribution of protons is obtained considering also the cooling through $p \gamma$ interactions with the low energy photons in the jet. These inelastic interactions, together with the $p p$ collisions, give rise to pions and their decay products, which include muons and neutrinos. The injection of pions $Q_{\pi}(z, E)$ can be worked out using the proton distribution $N_{p}(z, E)$. The distribution of pions $N_{\pi}(z, E)$ found with Eq. (2.5) is then considered to obtain the injection of muons $Q_{\mu}(z, E)$, which in turn is used to obtain the muon distribution $N_{\mu}(z, E)$. The different particle species are responsible for the radiative processes described in the next section.

\section{Radiative processes}

In the jet frame, where the particle distributions are isotropic, the synchrotron emissivity is

$$
Q_{\gamma, \text { syn }}^{\prime}\left(E_{\gamma}^{\prime}, z^{\prime}\right)=\frac{\epsilon_{\mathrm{syn}}^{\prime}\left(E_{\mathrm{ph}}^{\prime}, z^{\prime}\right)}{4 \pi E_{\gamma}^{\prime}},
$$

where $\varepsilon_{\text {syn }}^{\prime}$ is the power per unit energy per unit volume of the synchrotron photons,

$$
\varepsilon_{\mathrm{syn}}^{\prime}\left(E_{\mathrm{ph}}^{\prime}, z^{\prime}\right)=\left(\frac{1-e^{-\tau_{\mathrm{SSA}}\left(E_{\mathrm{ph}}^{\prime}, z^{\prime}\right)}}{\tau_{\mathrm{SSA}}\left(E_{\mathrm{ph}}^{\prime}, z^{\prime}\right)}\right) \int_{E_{e}^{\prime}}^{\infty} d E^{\prime} 4 \pi P_{\mathrm{syn}} N_{e}^{\prime}\left(E^{\prime}, z^{\prime}\right) .
$$

Here, $P_{\text {syn }}$ is the usual synchrotron power per unit energy emitted by the electrons.

The effect of synchrotron self-absorption (SSA) within the jet is determined by an optical depth

$$
\tau_{\mathrm{SSA}}\left(E_{\mathrm{ph}}^{\prime}, z^{\prime}\right)=\int_{0}^{\frac{r_{\mathrm{j}}\left(z^{\prime}\right)}{\sin \theta^{\prime}}} d l^{\prime} \alpha_{\mathrm{SSA}}\left(E_{\mathrm{ph}}^{\prime}, z^{\prime}, l^{\prime}\right) .
$$

$\theta^{\prime}$ is the viewing angle in the jet frame, and the SSA coefficient $\alpha_{\mathrm{SSA}}$ is evaluated with its standard expression along the path in the jet. The IC emissivity is

$$
Q_{\gamma, \mathrm{IC}}^{\prime}\left(E_{\gamma}^{\prime}, z^{\prime}\right)=\frac{r_{0}^{2} c}{2} \int_{\left.E^{\prime \prime \text { ph }}\right)}^{E_{\gamma}^{\prime}} d E_{\mathrm{ph}}^{\prime} \frac{n_{\mathrm{ph}}^{\prime}\left(E_{\mathrm{ph}}^{\prime}, z^{\prime}\right)}{E_{\mathrm{ph}}} \int_{E_{\mathrm{min}}^{\prime}}^{E_{\mathrm{max}}^{\prime}} d E^{\prime} \frac{N_{e}^{\prime}\left(E^{\prime}, z^{\prime}\right)}{\gamma_{e}^{2}} F(q) .
$$

The integration limits are:

$$
E_{\min }^{\prime}=\frac{E_{\gamma}^{\prime}}{2}+\frac{m_{e} c^{2}}{2} \sqrt{\frac{E_{\gamma}^{\prime}}{2 E_{\mathrm{ph}}^{\prime}}+\frac{E_{\gamma}^{\prime 2}}{2 m_{e}^{2} c^{4}}} \text { and } E_{\max }^{\prime}=\frac{E_{\gamma}^{\prime}}{1-\frac{E_{\gamma}^{\prime}}{E_{\mathrm{ph}}^{\prime}}} .
$$

As for the emission of protons, the relevant processes are $p p$ and $p \gamma$ interactions. The gamma-ray emissivity is

$$
Q_{\gamma, p p}^{\prime}\left(E_{\gamma}^{\prime}, z^{\prime}\right)=n_{\mathrm{c}}^{\prime}\left(z^{\prime}\right) c \int_{0}^{1} \frac{d x}{x} N_{p}^{\prime}\left(\frac{E_{\gamma}^{\prime}}{x}, z^{\prime}\right) F_{\gamma}\left(x, \frac{E_{\gamma}^{\prime}}{x}\right) \sigma_{p p}^{(\mathrm{inel})}\left(\frac{E_{\gamma}^{\prime}}{x}\right),
$$

where the function $F_{\gamma}\left(x, E^{\prime}\right)$ is the same as defined by Kelner et al. (2006), for a proton energy $E^{\prime}=E_{\gamma}^{\prime} / x$. The target-proton density in the jet frame is $n_{\mathrm{c}}^{\prime}\left(z^{\prime}\right)=n_{\mathrm{c}}\left(z^{\prime} \Gamma_{\mathrm{b}}\right) / \Gamma_{\mathrm{b}}$. Its value in the observer frame is

$$
n_{\mathrm{c}}(z)=\frac{\left(1-q_{\mathrm{rel}}\right) \dot{m}_{\mathrm{j}}}{m_{p} \pi z^{2} \tan ^{2} \xi_{\mathrm{j}} v_{\mathrm{b}}(z)} .
$$




\section{Spectral energy distributions}

The total luminosity $L_{\gamma}$ is calculated as

$$
L_{\gamma}=4 \pi \int_{V} d V E_{\gamma} Q_{\gamma}\left(E_{\gamma}, z\right) .
$$

where the photon emissivity $Q_{\gamma}\left(E_{\gamma}, z\right)$ corresponds to the observer frame.

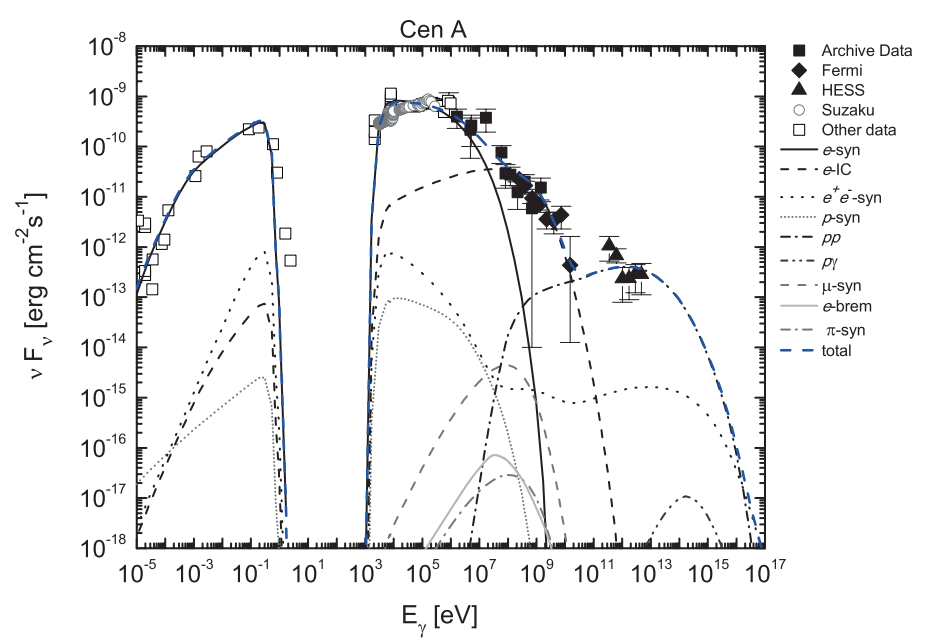

Figure 1. Lepto-hadronic model for the broadband spectral energy distribution of Centaurus A inner region.

The parameter $a=L_{p}^{\prime} / L_{e}^{\prime}$ takes the values $>>1$. The main contributions arise from synchrotron emission of electrons, inelastic $p p$ collisions and synchrotron radiation of secondary electron-positron pairs. The role of internal absorption is discussed by Reynoso et al. (2011). In Fig. 1 we show the application of the model to the available data for the inner region of Cen A.

The particle distributions described here correspond to a steady-state jet. Temporal variability can be introduced in different forms according to the timescales involved: 1 ) For long timescales (days or more), with a time-dependent injection $Q_{i}(z, E, t)$ and a time-derivative term $\partial N / \partial t$ in the transport equation. 2) For short timescales (hours or less), through the entrainment of magnetized clouds or stars in the jet as proposed by Araudo et al. (2010), or by bends or turbulence produced through instabilities (Romero 1995).

\section{Acknowledgments}

My work on this topic has been done in collaboration with M.M. Reynoso and M.C. Medina. I thank them both for fruitful discussions. This research was supported by CONICET (Argentina) through grant PIP 112-200901-00078.

\section{References}

Araudo, A. T., Bosch-Ramon, V., \& Romero, G. E. 2010, A\&A, 522, A97

Kelner, S. R., Aharonian, F. A., \& Bugayov, V. V. 2006, Phys. Rev. D, 74, 034018

Reynoso, M. M., Medina, M. C., \& Romero, G. E. 2011, A\&A, 531, A30

Romero, G. E. \& Vila, G. S. 2008, A\& $A, 485,623$

Romero, G. E. 1995, Astrophys. Sp. Sc., 234, 49

Vila, G. S. \& Romero, G. E. 2010, MNRAS, 403, 1457 